Recepción: 14 / 01 / 2018

Aceptación: 21 / 02 / 2018

Publicación: 21 / 04 / 2018

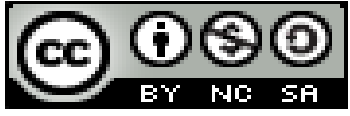

Ciencias sociales y políticas

Artículo de Revisión

\title{
La Intervención como experiencia profesional en Trabajo Social
}

\author{
Intervention as a professional experience in Social Work
}

\section{Intervenção como experiência profissional em Serviço Social}

\author{
Gustavo C. Solis-Mairongo I \\ gustavosolis1975@gmail.com \\ Maritza G. Espinoza-Cedeño ${ }^{\text {II }}$ \\ mary_espinoza86@ hotmail.es \\ Nube A. Muñoz-Zhañay III \\ alexandra_muñozz81@hotmail.com
}

Correspondencia: gustavosolis1975@gmail.com

I Magíster en Administración Pública Mención Evaluación de Proyectos, Licenciado en Trabajo Social, Docente de la Universidad Laica Eloy Alfaro de Manabí, Manta, Ecuador.

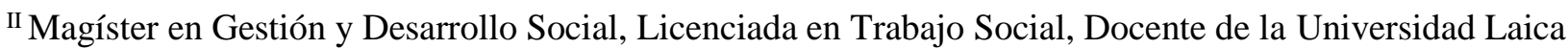
Eloy Alfaro de Manabí, Manta, Ecuador.

III Máster Universitario en Investigación e Intervención Psicosocial, Licenciada en Trabajo Social, Docente de la Universidad Laica Eloy Alfaro de Manabí, Manta, Ecuador. 


\section{Resumen}

El presente artículo es el resultado de un estudio llevado a cabo con diversos profesionales del Trabajo Social que desarrollan su labor en diferentes sectores de intervención. Es de interés para este estudio cómo se evalúa cada uno de los casos en los que los y las profesionales del Trabajo Social realizan día a día su labor profesional de intervención profesional. La intervención en Trabajo Social, sin duda, se complementa con las intervenciones de otros profesionistas; no sólo los trabajadores sociales intervienen profesionalmente. Es relevante hablar de la intervención de Trabajo Social como un proceso, en el que el hacer es esencia, momento que se construye en articulación con los momentos de antes y de después, centrando la atención en su contenido como un todo, como una unidad. Este trabajo sugiere que la experiencia del trabajador social en la intervención en el Trabajo Social, puede realmente contribuir de manera más determinante al cambio social que urge a la colectividad.

Palabras clave: Trabajo social; experiencia; valoración profesional; empoderamiento; intervención social.

\section{Abstract}

This article is the result of a study carried out with various Social Work professionals who work in different sectors of intervention. It is of interest for this study how each one of the cases in which the professionals of the Social Work perform every day their professional work of professional intervention is evaluated. The intervention in Social Work, without a doubt, is complemented with the interventions of other professionals; not only social workers intervene professionally. It is relevant to speak of the intervention of Social Work as a process, in which doing is essence, a moment that is constructed in articulation with the moments before and after, focusing attention on its content as a whole, as a unit. This work suggests that the experience of the social worker in the intervention in Social Work, can really contribute in a more decisive way to the social change that urges the community.

Keywords: Social work; experience; professional valuation; empowerment; social intervention.

\section{Resumo}

Este artigo é resultado de um estudo realizado com diversos profissionais do Serviço Social que atuam em diferentes setores de intervenção. É de interesse para este estudo como cada um dos 
casos em que os profissionais do Serviço Social realizam a cada dia o seu trabalho profissional de intervenção profissional é avaliado. A intervenção no Serviço Social, sem dúvida, é complementada com as intervenções de outros profissionais; não só os assistentes sociais intervêm profissionalmente. É relevante falar da intervenção do Serviço Social como um processo, em que fazer é essência, momento que se constrói em articulação com os momentos antes e depois, enfocando a atenção em seu conteúdo como um todo, como uma unidade. Este trabalho sugere que a experiência do assistente social na intervenção no Serviço Social, pode realmente contribuir de forma mais decisiva para a mudança social que instiga a comunidade.

Palavras chave: Trabalho social; experiência; avaliação profissional; empoderamento, intervenção social.

\section{Introducción}

Según el Diccionario de la Academia Española (2001), etimológicamente, el término "intervención" hace referencia a la acción y efecto de intervenir; la palabra "intervenir" significa tomar parte en un asunto y también interceder o mediar por alguien.

La intervención en el Trabajo Social, es la acción organizada y desarrollada por los trabajadores sociales con las personas, grupos y comunidades. Para la trabajadora social y profesora María Asunción Martínez Román (2003), dicha intervención está orientada a acompañar, ayudar y capacitar a las personas en sus procesos vitales para ser responsables, para ser libres de elegir y ejercer la participación, así como a facilitar los cambios de aquellas situaciones que supongan un obstáculo para el desarrollo humano y la justicia social, promoviendo los recursos de la política social, las respuestas innovadoras y la creación de recursos no convencionales, basados en el potencial solidario de las personas. Para tal fin, afirma la autora que no sólo se actúa profesionalmente con la gente y su ambiente más próximo, como es la familia, amigos y vecinos, sino que se intenta intervenir también en los contextos que condicionan o limitan sus posibilidades de desarrollo, teniendo como referentes la Declaración Internacional de los Derechos Humanos y la promoción de la justicia.

Barranco C. (2004), sostiene que la intervención en el Trabajo Social es entendida como la acción organizada y desarrollada por los trabajadores sociales con las personas, grupos y comunidades. Sus objetivos están orientados a superar los obstáculos que impiden avanzar en el desarrollo 
humano y en la mejora de la calidad de vida de la ciudadanía. Intervención profesional que se basa en los fundamentos éticos, epistemológicos y metodológicos del Trabajo Social, desde un enfoque global, plural y de calidad. Estos planteamientos del Trabajo Social parten de una concepción dinámica de la persona que se inscribe en un contexto social, histórico, político, económico y cultural de la realidad social, conectando con otras disciplinas y profesionales.

La experiencia en Trabajo Social, como argumenta Travi B. (1999), cuenta con un enorme potencial teórico/metodológico que por múltiples razones (internas y ajenas a la disciplina) no siempre llega plasmarse en la práctica cotidiana o en la reflexión teórica. Creemos que algunas de variadas las razones de esta situación, pueden estar vinculadas con:

- las condiciones laborales del ejercicio profesional;

- con la definición de los sujetos sociales "objetos de intervención";

- y con la histórica dificultad de articular la interpretación y la intervención lo cual se manifiesta, en algunos casos, en ciertas prácticas profesionales "rutinizadas' y en una escasa profundización o utilización poco rigurosa de categorías de análisis indispensables para el ejercicio profesional.

La intervención en Trabajo Social, se centra tanto en la persona como en el medio social, partiendo de un enfoque global y plural. Intervención profesional que se inscribe en un contexto social, histórico, político, económico y cultural de la realidad social. Esta parte de los principios éticos, epistemológicos y metodológicos de la profesión, basados en una concepción dinámica de la persona y del mundo. Los objetivos específicos y los modos concretos de llevar a cabo la intervención están supeditados a las características de la realidad de las personas y del contexto social, a los niveles y modelos de intervención del Trabajo Social desde los cuales se intervenga. (Barranco C., 2004). A partir de estas consideraciones previas sobre la disciplina y la profesión, vamos a señalar algunas de las características generales de la intervención en Trabajo Social, las cuales se representan en el siguiente dibujo. 


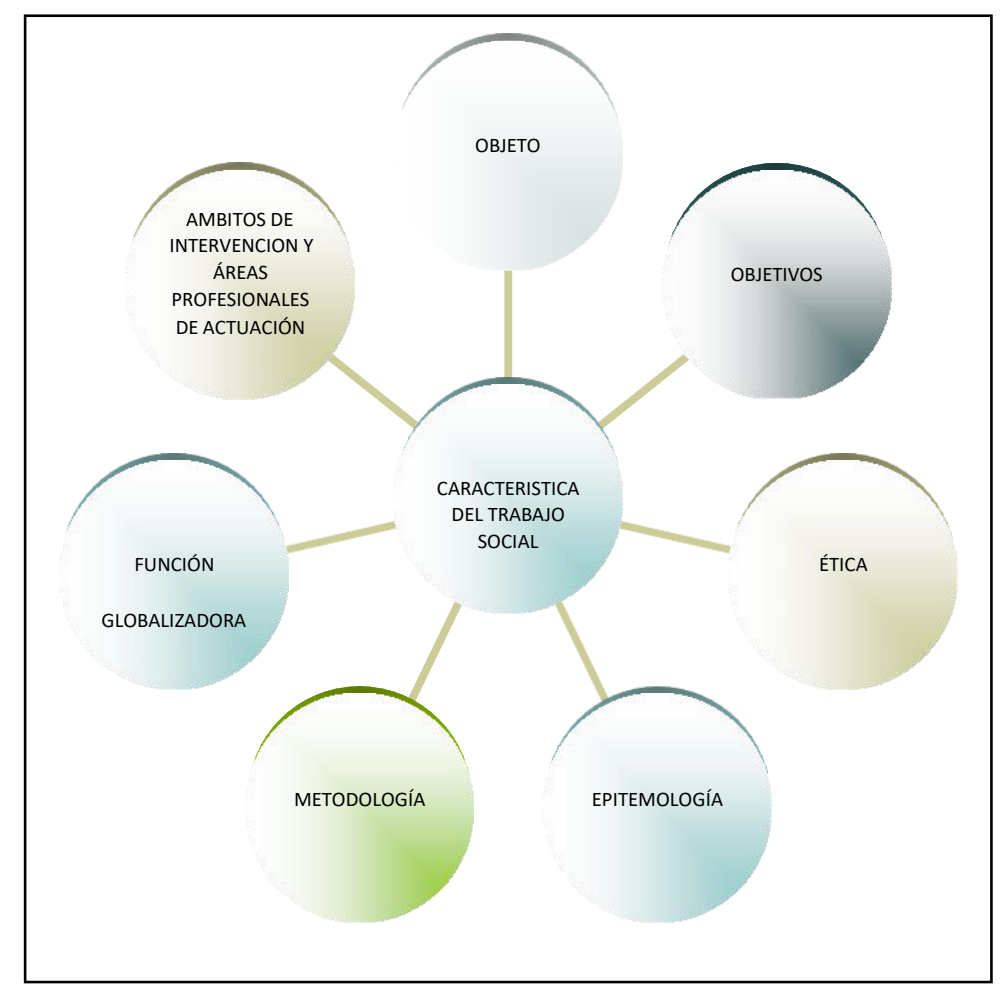

Gráfico 1. Características generales de la intervención en Trabajo Social

Fuente: Elaboración propia

\section{Desarrollo}

\section{Áreas profesionales y entornos de intervención}

Barranco (2004) explica que la intervención en Trabajo Social se lleva a cabo en los diversos ámbitos de los Servicios Sociales y de Bienestar Social, así como en otros ámbitos comunitarios, el socio sanitario, de Derechos Humanos, etc. En estos ámbitos de intervención profesional, los trabajadores sociales efectúan varias actividades que se compilan en las denominadas "áreas profesionales", siendo definida el área profesional como: "la agrupación de funciones profesionales relacionadas" (Consejo General de Colegios Oficiales de Diplomados en Trabajo Social y Asistentes Sociales de España, 2003, 17). A su vez, el área se define como "la capacidad de acción propia de los cargos y oficios" (Diccionario de la Real Academia Española, 2001). A manera de resumen se detallan algunas de las áreas más importantes, clasificadas por la Comisión Mixta del Perfil Profesional (2003). 
a. Área de atención. La intervención del Trabajo Social se concreta en las acciones con las personas y colectivos que sufren las consecuencias de situaciones carenciales, conflictos y crisis. Su fin es posibilitar el desarrollo humano y autonomía a través de la movilización de sus capacidades y de los recursos.

b. Área de prevención. La intervención profesional se centra en las causas de las dinámicas de marginación, exclusión y desintegración social, con la finalidad de evitar su aparición.

c. Área de promoción y educación. El Trabajo Social se orienta a descubrir y potenciar las capacidades de individuos, grupos y comunidades. También, a generar procesos de participación e implicación, de formación y de organización para ayudar a las personas a la adquisición o incremento de los conocimientos y habilidades sociales que precisan.

d. Área de mediación y arbitraje. A través de esta área los trabajadores sociales intervienen para mediar en la resolución de los conflictos que afectan a las familias y grupos sociales, tanto en el interior de sus relaciones, como en su entorno social

e. Áreas de planificación y evaluación. La intervención de los trabajadores sociales se desarrolla en el diseño de la planificación y de la evaluación de los planes, programas y proyectos.

f. Áreas de gerencia y administración. La intervención profesional se realiza en los procesos de organización, coordinación, dirección y animación del personal hacia la consecución de los objetivos y obtención de resultados de calidad.

Área de investigación y docencia. Los trabajadores sociales a través de la investigación tratan de profundizar en el conocimiento de las necesidades y problemas, así como en los procesos de calidad que se relacionan con su propio trabajo profesional. Al mismo tiempo, realizan acciones para que los conocimientos acumulados, las experiencias vividas y las reflexiones teóricas se pongan a disposición de los profesionales, de las personas vinculadas a la acción social y de los futuros trabajadores sociales.

\section{Tipos de intervención}

Vélez (2000), respecto a los tipos de intervención, plantea que en estos programas se hacen evidentes mediante la intención de satisfacer necesidades, de afrontar y solucionar rápidamente 
problemáticas ya consideradas, de favorecer gestiones que impulsan al desarrollo humano y la calidad de vida, evitando nuevos problemas que puedan deteriorar el libre desarrollo y la salud integral social e individual. La educación, además del aprendizaje de alfabetización, supone aprender a vivir en comunidad y a participar en la vida política, social y cultural. Detrás de estos procesos educativos están las intenciones de promocionar los espacios y los recursos de los cuales disponen los ciudadanos (y que ignoran), y además las intenciones de actuar anticipadamente ante posibles problemas sociales.

Se presentan los dos niveles de intervención reconocidos en Trabajo Social: directo e indirecto. Entre estos dos tipos se evidencia una tendencia hacia el nivel de intervención directo, que va muy de la mano con la preferencia que tienen los profesionales por el trabajo que implique el contacto con las personas. El nivel de intervención indirecto, aunque no es la tendencia, se hace presente en todos los procesos; pues aunque sea directa siempre es necesario planear, coordinar y cumplir con requerimientos, resultando impensable la intervención sin uno de estos niveles.

En los distintos procesos se distinguen diferentes objetos. Lo que le representa al profesional pensar estrategias metodológicas, referentes teóricos y conceptuales que propicien el encuentro con el otro en un espacio de reflexión y construcción para la trasformación, que no esté dado solo por los esfuerzos del profesional sino también de los sujetos de la intervención, que posibilite hablar de corresponsabilidad, como principio orientador de la intervención.

La intervención profesional debe contar con unas características innovadoras que respondan a las nuevas dinámicas sociales, y al objeto de la intervención. Donde se puedan seguir promoviendo sujetos gestores de su propio desarrollo y de nuevas ciudadanías, e incentivando proyectos orientados a un desarrollo más humano. Y donde lo público sea el espacio en que se configuran diferentes escenarios a la profesión para el ejercicio profesional; donde los profesionales actúan como dinamizadores de las propuestas de intervención articuladas a políticas públicas. (Mosquera A., y Ramírez B. 2009)

\section{La intervención profesional desde la experiencia del Trabajo Social}

La intervención profesional puede ser percibida como un proceso complejo que requiere de una constante reflexión crítica a partir de un diálogo entre los componentes, y el conocimiento producido alrededor de este. 
Según lo anterior, comprender, significar y re-significar la intervención profesional se logra en la articulación dialógica de los componentes, en consideración a las características de los diferentes escenarios desde donde el trabajador social interviene, y los objetivos que se plantean desde la práctica.

Los procesos de intervención en los que se insertan los profesionales son diferentes, y es indiscutible que desde cada proyecto y política social el profesional brinda características particulares a su intervención, que aportarán a la significación de esa intervención. En esta dirección, se trabajarán las categorías enunciadas en el referente conceptual y el sistema categorial, desde cuatro ejes, a saber:

- Eje epistemológico. Relacionado con la comprensión e interpretación que se hace de la realidad desde unas perspectivas de conocimiento, unas teorías y unos fundamentos. Esta presupone, según Yáñez (2007), una comprensión de la realidad, ofreciendo las bases para el pensamiento científico. Se ubican aquí como elementos claves la fundamentación teórica y conceptual, y el objeto de intervención y conocimiento.

- Eje ontológico. Tiene que ver con la naturaleza de la profesión y de la intervención. Permite comprender la existencia del trabajo social que lleva el ser en sí mismo, haciendo referencia a su origen, en cuanto a esencia y existencia disciplinaria. En este eje se ubica la concepción del otro y la identidad profesional, sin las cuales no se podría desarrollar el ejercicio profesional, en tanto los sujetos son los portadores de las situaciones a trasformar; y la identidad, si se puede decir así, es el punto de referencia de la actuación.

- Eje metodológico. Reflexiones que se realizan desde unos referentes teóricos y conceptuales que permiten la superación de la visión operativa e instrumental que se le ha dado, al reducirlo al método, donde se agrupan el método, las técnicas, los instrumentos, las funciones, los roles, los tipos y niveles de intervención; como aspectos claves para comprender lo metodológico en la práctica profesional.

- Eje ético-político. Es la reflexión que se realiza en razón de un ejercicio profesional consciente y el papel que el profesional desempeña. Además, se asume un compromiso a nivel profesional, así como social, al incidir en situaciones problemáticas, en la producción de conocimiento, y en la reflexión constante entre los componentes de la intervención y la 
realidad. En este sentido, se ubican las intencionalidades, principios, valores y apuestas profesionales.

En el análisis de la información de los componentes de la intervención, se identifica que los profesionales llenan de contenido cada una de las categorías, a partir de la reiterada asociación de los conocimientos que se adquieren en el proceso educativo, aspecto que se hace evidente en cada uno de los componentes de la intervención profesional, pues al profesional le resultaría imposible la configuración y comprensión de esta si no cuenta para ello con todo un acervo de conocimientos, desde los cuales se le posibilita hacer lecturas de la realidad y construir nuevos significados alrededor de la intervención profesional y sus componentes.

Los componentes de la intervención que convergen en el ejercicio profesional, la apuesta del trabajador social y la institución, tienen en algunos casos puntos de encuentro, dando lugar a que el profesional se identifique con el proceso.

Al articular los aportes que los trabajadores sociales realizan desde sus experiencias, y la reflexión que se ha venido planteando, se pueden identificar dos líneas del significado que, desde la interacción con la realidad y desde diferentes escenarios, se puede construir sobre la intervención profesional:

- La primera es de tipo funcional/operativa, donde el profesional cumple con unos requisitos y unos objetivos que se plantean desde la política social y desde el proyecto a ejecutar. Pues no hay una reflexión en cuanto a los componentes de la intervención, el papel, y el saber específico que los distingue de otros profesionales. En este sentido, se puede decir que no hay avances que le aporten a la conceptualización de la intervención profesional. Por lo contrario, se producen una serie de cuestionamientos frente a la apuesta ética y política del profesional, frente al autorreconocimiento de la especificidad, de los roles y funciones como profesional del Trabajo Social.

- La segunda es de tipo interpretativa/reflexiva, donde los profesionales, además de cumplir con los requerimientos por los cuales se les contratan, se cuestionan permanentemente su ejercicio y la puesta en escena de lo público (el saber específico de la profesión). Desde allí podrá hacer aportes a la transformación no solo de la realidad concreta sobre la que se interviene, sino que también puede aportar a la construcción de las políticas públicas y leyes (en el orden municipal, 
departamental y nacional) que propendan por el bienestar de la población más vulnerable. (Mosquera A., y Ramírez B. 2009)

Identificar los significados que se realizan, puede evidenciar que en algunos no hay avances en la conceptualización del ejercicio profesional. Se hacen notorios los retrocesos conceptuales. Se habla de retrocesos, en la medida que, en la articulación de conocimientos y construcción de nuevos significados, se desdibujan algunos aspectos del saber específico de la profesión.

\section{Conclusiones}

Es posible hablar de un significado, o significados, de la intervención profesional. Se habla de significado, en tanto se hace una elaboración de este, considerando los aportes que desde cada experiencia se posibilita sostener para la construcción de un significado objetivo.

Para dimensionar el significado real que los trabajadores sociales le han dado a la intervención profesional, como ya se había señalado, es oportuno que esta categoría científica y sus componentes sean vistos y comprendidos desde el escenario de lo social; en el contexto de la práctica y en la dinámica de la política social, pues es allí donde se evidencia cómo ha sido entendida, significada y re-significada.

En ese sentido, la intervención profesional, según las experiencias de los trabajadores sociales, es entendida como un proceso de construcción y trasformación de lo social, como producto de las interacciones que se dan en un escenario y contexto determinados; razón por la que resultaría impensable una construcción del significado del concepto sin considerar las interacciones y las vivencias de los profesionales. Es decir, esta construcción es el resultado de las interacciones y las reflexiones que se generan en torno a la intervención profesional, sus categorías y subcategorías; en este sentido, no consiste en el diseño, implementación u operativización de estrategias.

\section{Referencias Bibliográficas}

BARRANCO, C. (1999). Estudio de los CAMPs de Tenerife. Tesis doctoral. La Laguna: Universidad de la Laguna, Departamento de Didáctica y Ciencias del Comportamiento. 
BARRANCO, C. (2002a). La Calidad percibida en Centros de Atención a Personas con Discapacidad Psíquica de Tenerife. Un enfoque integrado de la Calidad de Vida, Calidad de Servicio y Calidad de Vida Laboral. Tenerife: Cabildo de Tenerife.

BARRANCO, C. (2002b). Calidad de viday Trabajo Social. Planteamientos teóricos y experiencias en organizaciones de bienestar social y en comunidades. AGATHOS, 4, 4-21.

BARRANCO, C. (2004) Los profesionales de ayuda y el burnout. Trabajo Social y Salud, 47, 2737.

BARRANCO, C. (2004). La intervención en trabajo social desde la calidad integrada. Alternativas: Cuadernos de trabajo social. 12 (4), págs. 79-102.

BARRANCO, C; CÁCERES, C; CÍVICOS, A.; DÍAZ VILELA, F. L.; HERNÁNDEZ

HERNÁNDEZ, M. y PUYOL, B. (2003). Informe de la Evaluación de la Calidad Servicio de los Centros Ocupacionales de la Provincia de Santa Cruz Tenerife. Tenerife: Universidad de La Laguna. Departamento de Trabajo Social y Servicios Sociales.

BARRIGA, L. A. (2000). Calidad en los servicios sociales reflexiones sobre la calidez total. Revista de Servicios Sociales y Política Social, 49, 31-62.

COMISIÓN MIXTA DEL PERFIL PROFESIONAL (2003). Perfil Profesional del Trabajador Social Siglo XXI. Madrid: documento interno.

COMUNIDAD DE MADRID (1999). Satisfacción de los ciudadanos y ciudadanas. Madrid: Dirección General de Calidad de los Servicios.

\section{CONSEJO GENERAL DE COLEGIOS OFICIALES DE DIPLOMADOS EN TRABAJO}

SOCIAL Y ASISTENTES SOCIALES (1999). La ética del trabajo social principios y criterios. Recuperado el 14 de abril de 1999, de htpp://www.cgtrabajosocial.es/

\section{CONSEJO GENERAL DE COLEGIOS OFICIALES DE DIPLOMADOS EN TRABAJO}

SOCIAL Y ASISTENTES Sociales de España, (2003). Perfil profesional trabajador social siglo XXI (borrador). Madrid: documento inédito. CROSBY, P.B. (1979): Quality is Free. Nueva York, MacGraw-Hill. 
DEMING, E.W. (1981): Management and Stadistical Techniques for Quality and Productivity. New York. New York University (School Business).

FERNÁNDEZ-BALLESTEROS, R.; ZAMARRÓN, M.D. y MACÍA, A. (1996). Calidad de vida en la vejez en distintos contextos. Madrid: INSERSO.

FEIGENBAUM, A.V. (1984): «Product quality: how t o get it; how to keep it». Chief Executive, $\mathrm{n}^{\circ} 27$, págs. $20-23$.

FUNDACIÓN CANARIA DE INVESTIGACIÓN Y SALUD (2000). Cuestionario EuroQol. Tenerife: FUNCIS.

\section{FUNDACIÓN EUROPEA PARA LA MEJORA DE LAS CONDICIONES DE VIDA Y} TRABAJO (2002). Seminario: La calidad de los servicios sociales públicos. Resultados y conclu102 Carmen Barranco Expósito siones. Irlanda: Fundación Europea para la Mejora de las Condiciones de Vida y de Trabajo. Recuperado el 15 de mayo de 2003, de http://www.eurofound.eu.int.

GIL-MONTE, P. (1994): Tesis Doctoral. La Laguna: Universidad de La Laguna.

GUILLEN, E. y PÉREZ, D. (2002). Procesos de mejora: calidad, decisiones e innovación. En T. Fernández y A. Ares (Coords.). Servicios Sociales: Dirección, gestión y Planificación. Madrid: Alianza.

GUTIÉRREZ RESA, A. (2002). Motivación en los Servicios Sociales. En T. Fernández y A. Ares (Coords.). Servicios Sociales: Dirección, gestión y Planificación. Madrid: Alianza.

HOWE, D. (1999). Dando sentido a la práctica. Una introducción a la teoría del Trabajo Social. Granada: Maristan. (Orig. 1992).

JURAN, J.M. (1982): UpperManagemenetandQuality. NuevaYork, Juran Institute.

LÓPEZ, J. y GADEA, A. (1995). Servir al ciudadano. Gestión de la Calidad en la Administración Pública. Barcelona: Ediciones Gestión 2000.

LEVI, L. y ANDERSON, L. (1980): La tensión psicosocial. Población, ambiente y calidad de vida. México D. F., El Manual Moderno. 
MARTÍNEZ ROMÁN, M.A. (2003). Consideraciones generales: relaciones del Trabajo Social con el bienestar social, Estado de bienestar, política social, servicios sociales, diferenciación de conceptos.

MOSQUERA A., Y RAMÍREZ B. (2009). Significando la intervención profesional desde la experiencia de trabajo social. Revista Trabajo Social N. ${ }^{\circ}$ 15, enero-junio, 2012 pp. 33-54.

VERDUGO, M.A. (2003). Calidad de vida. Manual para profesionales de la educación, salud y servicios sociales. Madrid: Alianza.

RED, N. DE LA, (1996). Epistemología y Metodología de Trabajo Social. En / Congreso Estatal de Escuelas Universitarias de Trabajo Social. Valencia: Apuntes de la ponencia.

RED, N. DE LA, (2000). La Metodología en el Trabajo Social desde el marco de las ciencias sociales. CABS. Cuadernos Andaluces de Bienestar Social, 8, 9-32.

TRABI BIVIANA (1999). REFLEXIONES EN TORNO A LA INTERVENCiÓN PROFESIONAL DESDE EL TRABAJO SOCIAL CON MUJERES PERTENECIENTES A

LOS SECTORES POPULARES. La Aljaba, segunda época, 6 (129). Disponible en: http://www.biblioteca.unlpam.edu.ar/pubpdf/aljaba/v04a09travi.pdf

VÉLEZ RESTREPO, OLGA LUCÍA (2000): Perspectivas del Trabajo Social en el siglo xxi. En Revista Colombiana de Trabajo Social, Conest, N. ${ }^{\circ}$ 14, marzo

YÁÑEZ PEREIRA, VÍCTOR RODRIGO (2007): Visibilidad/invisibilidad en el Trabajo Social. Los fundamentos de una cosmología disciplinar. Buenos Aires: Espacio. 